\title{
Acute mesenteroaxial gastric volvulus on computed tomography
}

\author{
A Ahmed \\ Visser, Erasmus, Vawda \& Partners, Port Elizabeth \\ A Ahmed, MB BCh, FCRad (Diag) \\ Corresponding author: A Ahmed (docaadil@gmail.com)
}

\begin{abstract}
Acute gastric volvulus is a rare, but potentially life-threatening, cause of upper gastro-intestinal obstruction. The diagnosis can prove clinically challenging, and hence there is increased reliance on imaging. There are different types of gastric volvulus, with the variant presented in our case being the less commonly encountered mesenteroaxial gastric volvulus. Some of the CT features of gastric volvulus are described, and the usefulness of CT in assisting with the diagnosis is highlighted.
\end{abstract}

S Afr J Rad 2013;17(1):21-23. DOI:10.7196/SAJR.817

Gastric volvulus is a rare clinical entity, and a clinically relevant cause of acute abdominal pain in adults. It may prove to be a diagnostic dilemma for clinicians in view of the nonspecific clinical symptoms. The imaging diagnosis also remains challenging. ${ }^{[1]}$

Abdominal computed tomography (CT) has been underutilised in the diagnosis of gastric volvulus in previously reported series. ${ }^{[2]}$ The present case illustrates the value and importance of abdominal CT in the rapid diagnosis of acute abdominal pathology and, in particular, acute gastric volvulus. It is imperative that the diagnosis is made early in the course of the disease, to allow prompt surgical intervention and prevention of life-threatening complications.

\section{Case presentation}

A 41-year-old woman presented to the emergency department with a 1-day history of severe upper abdominal pain and nausea. Examination revealed features of an acute abdomen with the clinical suspicion of a bowel perforation. She had no other gastro-intestinal tract symptoms. She was not on any chronic medication and had no history of prior abdominal surgery or trauma.

In view of the acute but non-specific symptoms, a CT of the abdomen was requested. No oral or IV contrast was administered in view of the clinical condition of the patient and the diagnosis being evident on the initial uncontrasted scan. The patient was taken for emergency surgery soon thereafter. The diagnosis of mesenteroaxial gastric volvulus was confirmed; fortunately there were no signs of ischaemia, necrosis or perforation. The patient had an uneventful recovery.

CT demonstrated an unusually high-positioned stomach with loss of the normal gastric axis and appearance. The axial set of images in Fig. 1 demonstrates this unusual-appearing stomach with the pylorus and gastro-oesophageal (GO) junction closely approximated to each other. The pylorus and antrum are positioned superior to the gastric fundus. The stomach had an abnormal transverse orientation, best demonstrated on the sagittal reformatted images (Fig. 2). Gastric folds that form secondary to twisting of the stomach are also seen on the axial and coronal images (Fig. 3). The spleen is also displaced inferiorly, in keeping with the described 'wandering spleen' (Fig. 3).

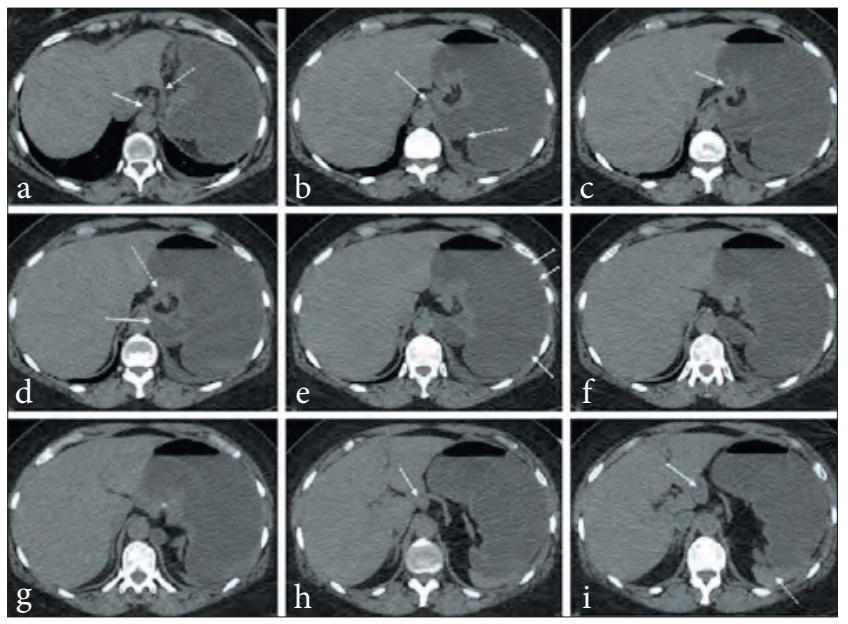

Fig. 1. Axial series of images through the abnormally orientated, distended stomach, progressing caudally from image A to I. (a) Distal oesophagus (solid arrow), gastrohepatic ligament (broken arrow). (b)GO junction (solid arrow), gastric fold (broken arrow). (c) Pylorus (solid arrow). (d) GO junction/ gastric cardia (solid arrow), gastroduodenal junction (broken arrow). The GO junction and gastroduodenal junction are closely approximated. (e) Greater curvature of the stomach (arrows). The greater and lesser curvatures maintain their normal relationship. (f) GO junction (arrow). (g) Gastric suspensory ligament (arrow). (h) First part of the duodenum (arrow), hepatoduodenal ligament. (i) Duodenum (solid arrow), displaced spleen (broken arrow).

No diaphragmatic hernia/defect or pneumoperitoneum was present. No additional abnormalities were seen on the CT examination of the abdomen.

\section{Discussion}

Gastric volvulus is defined as an abnormal, acquired rotation of the stomach of more than 180 degrees, creating a closed loop obstruction. Acute gastric volvulus is less common and is an acute, life-threatening, surgical emergency. If not recognised and treated quickly, it can result in complications such as gastric ischaemia, necrosis and perforation. ${ }^{[3]}$ 


\section{CASE REPORT}

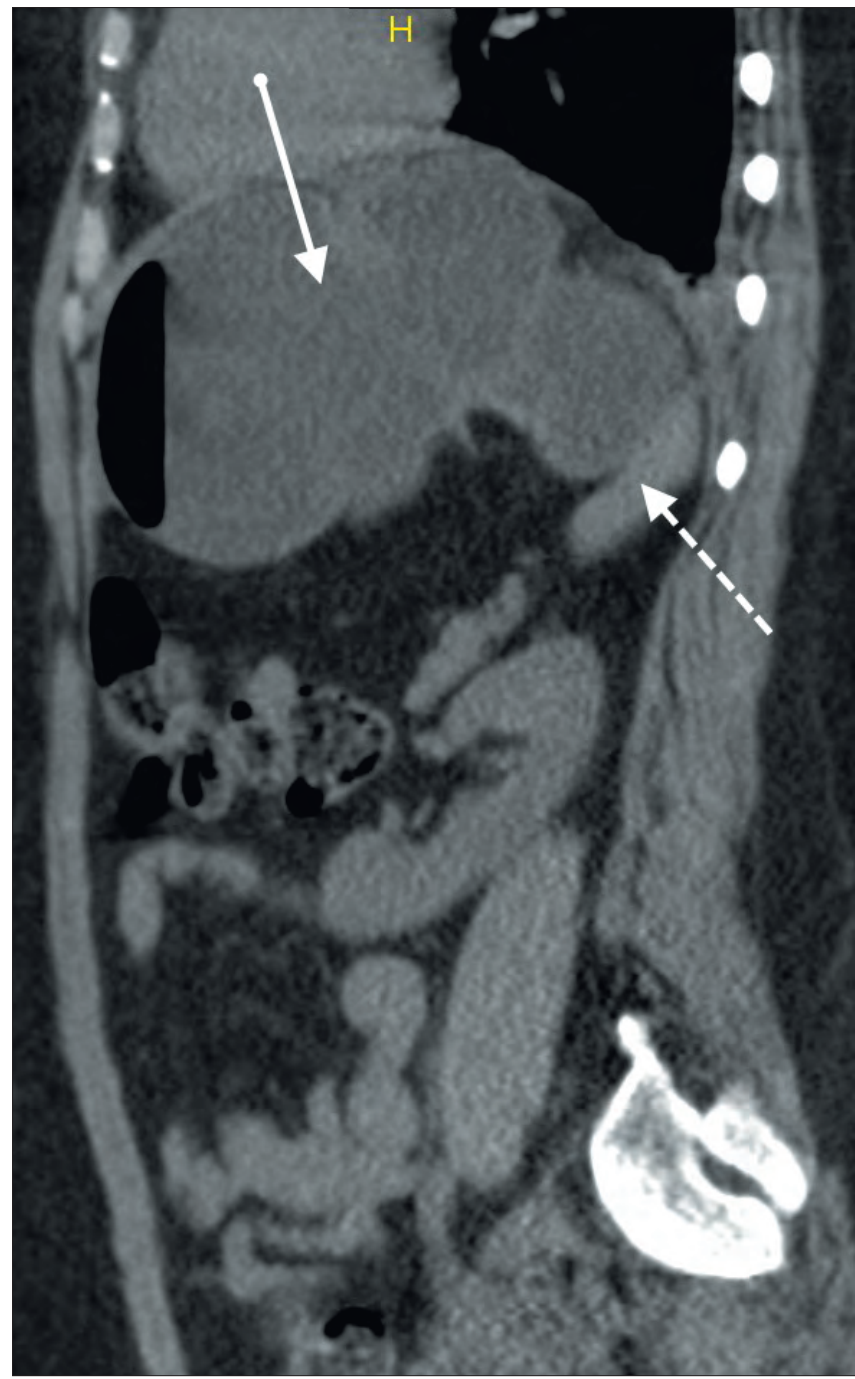

Fig. 2. Sagittal CT image demonstrating the abnormal horizontal orientation of the stomach (solid arrow). The spleen is indicated (broken arrow).

The Borchardt clinical triad of symptoms (epigastric pain, retching and inability to pass a nasogastric tube) are useful and valuable pointers to the suspected diagnosis. ${ }^{[1,4,5]}$

Gastric volvulus can be classified based on axis of rotation, severity (acute or chronic), extent (total or partial) or aetiology (primary or idiopathic). ${ }^{[6]}$ The most frequently used classification system describes 3 types of gastric volvulus: organoaxial, mesenteroaxial and combination-unclassified. ${ }^{[5,6]}$ This is based on the axis of rotation. Organoaxial is the most common variant, accounting for approximately two-thirds of cases, and occurs when the stomach rotates along its long axis (Fig. 4). ${ }^{[1,6]}$ The greater curvature is displaced superiorly, and the lesser curvature lies more caudally in the abdomen. If the twist is $>180^{\circ}$, gastric outlet obstruction occurs. The GO junction and pylorus maintain their normal anatomical positions and are not in close proximity.

There is also less risk of ischaemia in organoaxial volvulus. ${ }^{[7]}$ One of the predisposing factors for volvulus is the presence of a diaphragmatic defect; in adults, organoaxial volvulus occurs commonly in the setting of a post-traumatic diaphragmatic defect or a para-oesophageal hernia. ${ }^{[1,6]}$

Another predisposing factor for volvulus is the presence of an abnormality relating to the anchoring of the stomach and the

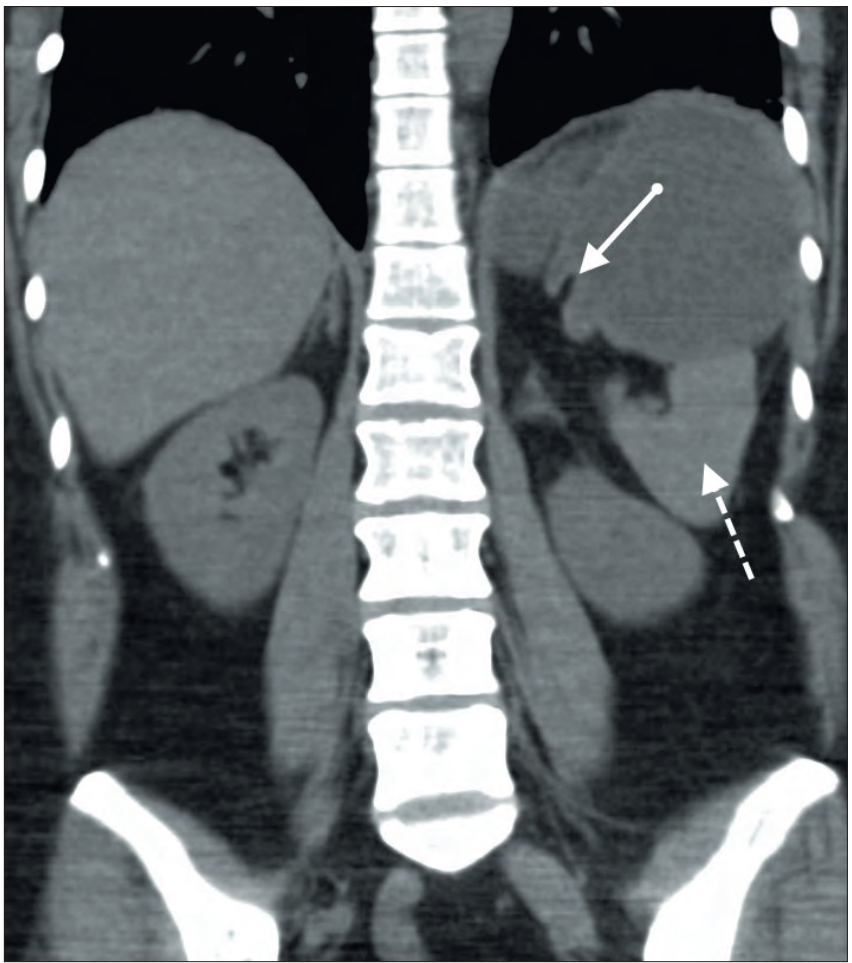

Fig. 3. Coronal CT image showing abnormal gastric fold (solid arrow). The inferiorly displaced 'wandering spleen' is also seen (broken arrow).

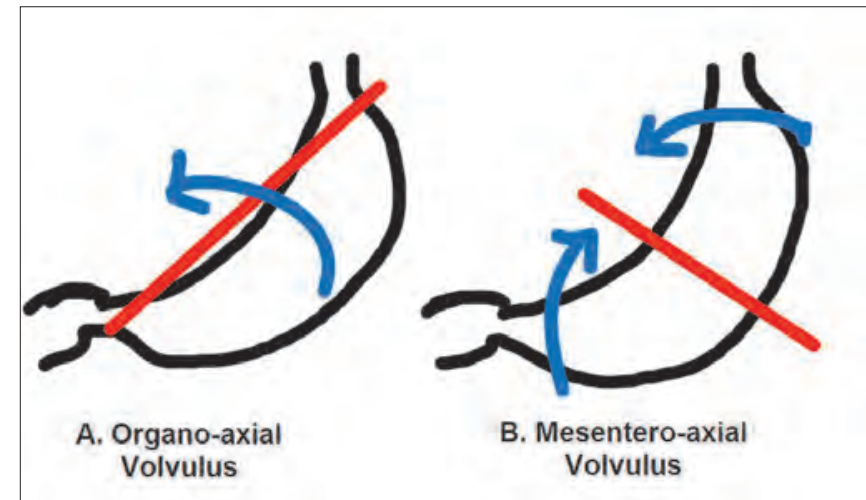

Fig. 4. Sketch demonstrating the mechanism of both (a) organoaxial and (b) mesenteroaxial volvulus. In (a), organoaxial volvulus develops secondary to twisting of the stomach along its long axis (red line). In (b), there is rotation of the stomach around the short axis (red line) resulting in mesenteroaxial volvulus.

surrounding ligaments. ${ }^{[6]}$ This is thought to be one predisposing factor in the development of the second type of volvulus, and the variant encountered in our patient - mesenteroaxial volvulus. The gastrohepatic ligament is demonstrated on the coronal and axial images (Fig. 1A). An unusually long gastro-hepatic mesentery has been mentioned as a predisposing factor for the development of mesenteroaxial gastric volvulus. ${ }^{[8]}$ Mesenteroaxial volvulus is much less common, accounting for approximately $29 \%$ of cases, and occurs when the stomach rotates around the transgastric/short axis (a line connecting the middle of the lesser curvature with the middle of the greater curvature) (Fig. 4). ${ }^{[6]}$ The anterior gastric wall flips on itself and the antrum is displaced above the GO junction. The greater and lesser curvatures are in their usual positions relative to each other. ${ }^{[7]}$ There is reversal of the relationship of the GO junction and pylorus, and they may lie in close 


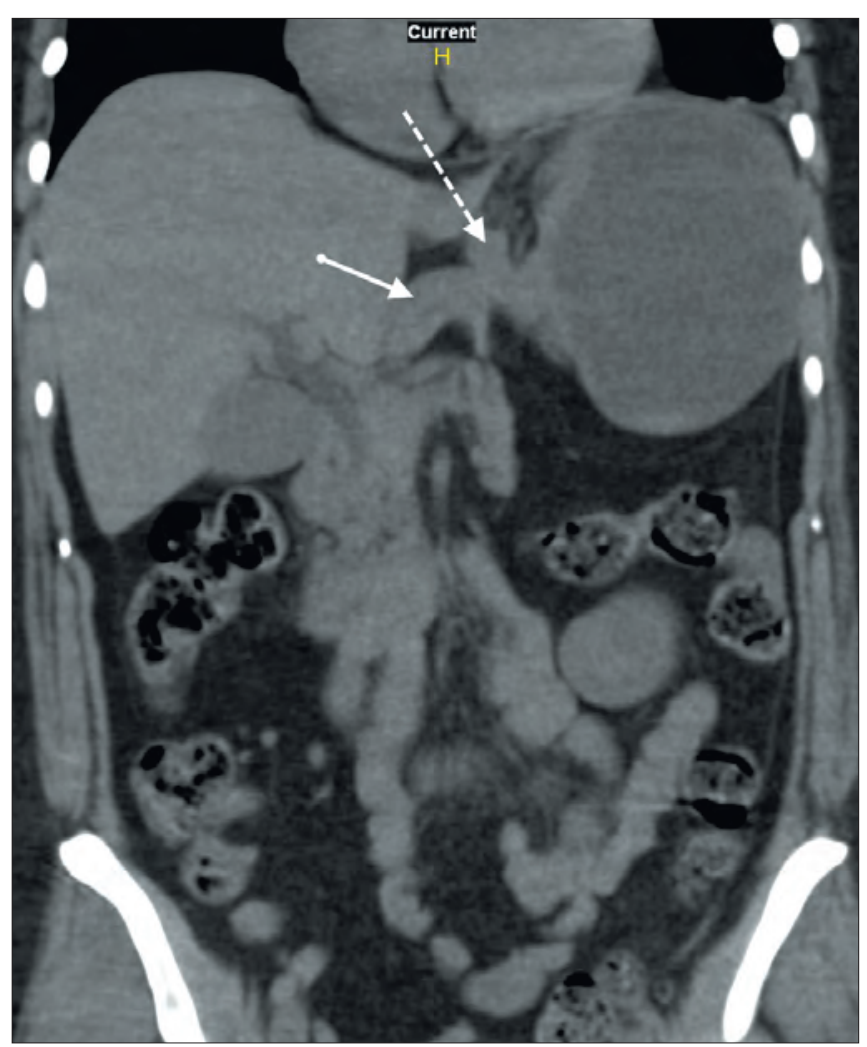

Fig. 5. Coronal CT image demonstrating the close relationship of the GO junction (broken arrow) and gastroduodenal junction secondary to the volvulus. The first part of the duodenum is shown by the solid arrow.

proximity to each other (Fig. 1); this creates a tapered pedicle about which the stomach can twist, making gastric ischaemia more likely in mesenteroaxial volvulus.

The third type of volvulus is the combined type that exhibits features of both mesenteroaxial and organoaxial volvulus. ${ }^{[3,5,6]}$ Mesenteroaxial and the combined type tend to present acutely. ${ }^{[3,7]}$

\section{Imaging}

Gastric volvulus may have a nonspecific clinical presentation, hence increasing the reliance on imaging to assist in coming to the correct diagnosis. It is also imperative that, in the acute setting, imaging studies do not result in delays in coming to a diagnosis and subsequent management. ${ }^{[3]}$ Radiography, upper gastro-intestinal tract contrast studies and CT are the imaging modalities most often used to make the diagnosis of volvulus. CT has been underutilised in previously reported series of gastric volvulus, and has not been advocated as the primary imaging study. There are reports in the literature demonstrating use of $\mathrm{CT}$ in the diagnosis of gastric volvulus. The acutely ill patient may also not tolerate oral contrast for the fluoroscopic examination, making CT a viable alternative.

In our patient, the CT scan was performed early, and expedited diagnosis and management. Abdominal CT is often done in the setting of abdominal pain and vomiting and can help in confirming abnormal rotation of the stomach. ${ }^{[1]}$ CT findings of a stomach that has an unusually high position, an abnormal axis, or an abnormal GO junction and pylorus relationship, should raise suspicion of gastric volvulus (Figs 1 and 5). ${ }^{[6,8]}$ The multi-planar reconstructions facilitate display of torsion of the stomach in various planes, assisting in confirming the diagnosis (Figs 2 and 5). The above-mentioned close approximation of the GO junction and pylorus, and the abnormal antral folds that can develop secondary to the twisting, are best seen on coronal images (Figs 3 and 5). The wandering spleen is frequently associated with gastric volvulus and is easily identified on CT (Fig. 3); ${ }^{[3]}$ this is secondary to absence of a ligamentous connection between the stomach and the spleen. ${ }^{\left[{ }^{[8]}\right.}$

There are further advantages that CT has over other imaging modalities:

1. detection of gastric pneumatosis and pneumoperitoneum, suggestive of necrosis and perforation, respectively

2. detection of predisposing factors, e.g. diaphragmatic defects or hernias, dense adhesions

3. detection of other abnormalities associated with gastric volvulus, viz. wandering spleen, intrathoracic kidney, malrotation with asplenia

4. excluding other extra-gastric or vascular causes of gastric ischaemia

5. detection of other causes of abdominal pain in the absence of gastric volvulus. ${ }^{[2,6]}$

Occasionally, the findings may be equivocal on CT. In such cases, an upper gastro-intestinal tract contrast study may be performed for confirmation. Chronic or intermittent volvulus (predominantly the organoaxial variant), which accounts for two-thirds of adult cases, may be asymptomatic and may be reported as an incidental finding on routine imaging studies. ${ }^{[5]}$ Importantly, CT scans performed in the asymptomatic interval ('untwisted' state) may miss the diagnosis completely. ${ }^{[2]}$

\section{Conclusion}

Acute gastric volvulus is an uncommon, and often unrecognised, surgical emergency that should be considered in patients who present to the emergency department with severe epigastric pain and evidence of gastric outlet obstruction. If the diagnosis is in doubt (and this is often the case), imaging studies are important. This case report highlights the usefulness of an abdominal CT in assisting in diagnosing this lifethreatening condition. The $\mathrm{CT}$ allows for multi-planar demonstration of abnormal torsion of the stomach and also provides valuable insights into possible aetiologies, and predisposing factors. CT is readily available nowadays, and should be considered the diagnostic tool of choice in any suspected gastric volvulus.

1. Peterson MC, Anderson JS, Hara AK, et al. Volvulus of the gastrointestinal tract: Appearances at multimodality imaging. Radiographics 2009;29:1281-1293. [http://dx.doi.org/10.1148/rg.295095011]

2. Woon CY, Chung AY, Low AS, Wong W. Delayed diagnosis of intermittent mesenteroaxial volvulus of the stomach by CT: A case report. Journal of Medical Case Reports 2008;2:343. [http://dx.doi. org/10.1186/1752-1947-2-343]

3. Casella V, Avitabile G, Segreto S, Mainenti PP. CT findings in a mixed-type acute gastric volvulus. Emergency Radiol 2011;18:483-486. [http://dx.doi.org/10.1007/s10140-011-0963-6]

4. Chandrasekhar C, Khanejaa S, Banullab P, Scheina M. CT diagnosis of acute gastric volvulus. Dig Surg 2003;20:497-499. [http://dx.doi.org/10.1159/000073536]

5. Van der Merwe DJ, Louw HB, Dekker G. Gastric volvulus - an explanation through imaging. S Afr J Radiol 2007;11(4):105.

6. Sevcik WE, Steiner IP. Acute gastric volvulus: case report and review of the literature. CJEM 1999;1:200203.

7. Oh SK, Hon BK, Levin TL, et al. Gastric volvulus in children: The twists and turns of an unusual entity. Pediatr Radiol 2008;38(3):297-304. [http://dx.doi.org/10.1007/s00247-007-0709-5]

8. Dahnert W. Radiology Review Manual. 7th ed. Philadelphia: Lippincott Williams \& Wilkins, 2011:852853. 